\title{
Dynamic Intermediates in the Radical Cation Diels-Alder Cycloaddition: Lifetime and Suprafacial Stereoselectivity
}

\author{
Jacqueline S. J. Tan, ${ }^{a}$ Viivi Hirvonen ${ }^{\mathrm{a}}$ and Robert S. Paton ${ }^{\mathrm{a}, \mathrm{b} *}$ \\ a Physical and Theoretical Chemistry Laboratory, University of Oxford, South Parks Road, Oxford OX1 3QZ, UK \\ ${ }^{\mathrm{b}}$ Department of Chemistry, Colorado State University, Fort Collins, Colorado 80523, USA
}

\begin{abstract}
Cation Radical Diels-Alder Cycloadditions proceed via an acyclic intermediate that exists on a flat region of the potential energy surface. Competition between cyclization and $\mathrm{C}-\mathrm{C}$ bond rotation results in varying levels of suprafacial stereoselectivity. Quasi-classical trajectories were used to explore reaction dynamics on this surface. Even though there is no discernible energy barrier towards cyclization, a dynamically stepwise process is found, for which the acyclic intermediate is found to reside for several hundreds of femtoseconds. In a small number of cases, exceptionally long lifetimes (>1000 fs) are found, leading to a loss of alkene stereochemistry.
\end{abstract}

Efficient thermal Diels-Alder $[4+2]$ cycloadditions typically require diene and dienophile components to be electronically matched. Conventionally, an electron-rich diene and an electron-poor dienophile are used. In contrast, the radical cationic Diels-Alder (DA) cycloaddition of electronically mismatched partners is possible due to much higher levels of reactivity. ${ }^{1-6}$ The [4+2] cycloadditions of electron-rich dienes with electronrich dienophiles showing poor conversion under neutral conditions have been successfully performed by radical cation generation using photochemical or chemical means. ${ }^{7,8}$ Additionally, radical-cation DA cycloadducts may show complementary regioselectivities to those obtained from corresponding neutral reactions. ${ }^{9,10}$

The degree of concert between the formation of both $\mathrm{C}-\mathrm{C}$ bonds remains a contentious issue for radical cation DA reactions. Seminal theoretical contributions from Wiest ${ }^{11}$ have established that the synchronous $\mathrm{C}_{\mathrm{s}^{-}}$ symmetric transition structure is unstable towards a pseudo-Jahn-Teller distortion, lying considerably higher in energy $(19 \mathrm{kcal} / \mathrm{mol})$ than the stepwise pathways. Nevertheless, differences in levels of suprafacial stereoselectivity have prompted mechanistic speculation on whether both stepwise and concerted pathways can take place (Scheme 1).

Scheme 1. Cation radical Diels-Alder cycloadditions of cis- and trans-anethole to cyclopenta-1,3-diene.

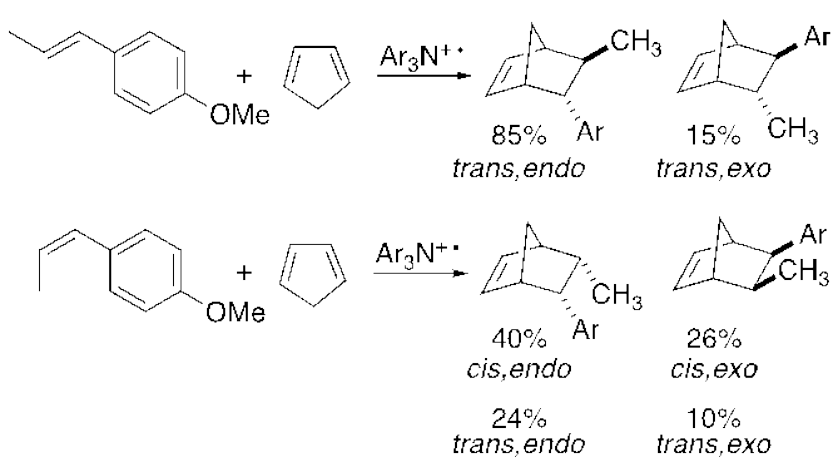

In the radical cation DA reactions of cyclopentadiene and anethole, Bauld observed exclusive formation of the trans-adducts from trans-anethole, whereas the cisdiastereomer gives a mixture of all four cis and transcycloadducts. ${ }^{12,13}$ In terms of a stepwise mechanism, these outcomes are consistent with the second $\mathrm{C}-\mathrm{C}$ bond forming step (i.e. ring-closure) being much faster (1000-fold) than bond rotation for trans-alkene, whereas bond rotation becomes competitive for cis-alkene. Alternatively, the (less-likely) idea of a mechanistic continuum has been proposed, in which trans- and cisisomers react via concerted and stepwise pathways, respectively. ${ }^{13}$

Understanding competition between bond rotation and cyclization in the radical cation DA reaction is key to addressing these stereochemical conundrums. This question is not one that can be easily addressed within the framework of transition state theory (TST), formulated by Eyring in the 1930s. The assumption that excess energy is distributed equally between all vibrational modes, faster than any chemical event is not necessarily true on a flat potential energy surface, such 
as that which characterizes the radical cation DA reaction. ${ }^{11}$ Another fundamental assumption behind TST is that each molecule crosses any TS only once, which is not always the case due to recrossing, although such recrossing can be minimized with variational TST. ${ }^{14-16}$ For this reason, recent attention has focused on the role of dynamics in reactions with shallow intermediates. ${ }^{17,18}$ Quasi-classical dynamics calculations have become a powerful tool in understanding selectivity for case which cannot be accurately (or at times, even at all!) predicted with TST. ${ }^{19-22}$ Houk has used these methods to shed light on the mechanism and synchronicity of Diels-Alder and ambimodal $[6+4] /[4+2]$ cycloadditions. ${ }^{23-26}$

To explore the degree of concerted and stereoselectivity in the radical cation Diels-Alder reaction, we have used SMD-M06-2X/6-31G(d) density functional theory (DFT) calculations to characterize the potential energy surface (PES) and to perform quasiclassical dynamics simulations. The extreme flatness of this PES, and low barrier pathways for several competing reactions make this an ideal candidate for such a study. For the exemplar reaction of ethylene and cyclopentadiene, we focus on events following the dynamic bottleneck, to understand the competition between $\mathrm{C}-\mathrm{C}$ rotation and the formation of cycloadducts.

Scheme 2. (a) Concerted, neutral DA and (b) stepwise, radical cation DA reaction of ethylene and cyclopentadiene are shown at SMD-M06-2X/6-31G(d) level with Gibbs energies quoted in $\mathrm{kcal} / \mathrm{mol}$.

(a)

(b)

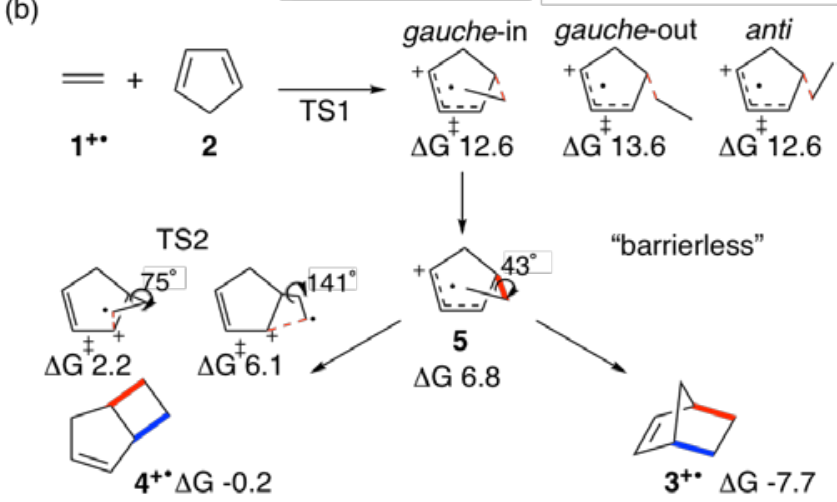

reaction occurs in a stepwise fashion, with the intervention of an acyclic intermediate $5^{+}$. The overall barrier is much lower than the neutral reaction. Cyclization of this intermediate can occur to form the DA adduct $\mathbf{3}^{+}$. or four-membered bicyclo[3.2.0]hept-2-ene $4^{+}$. We located three TSs on the potential energy surface (PES) corresponding to the first elementary step, which differ by rotation about the forming $\mathrm{C}-\mathrm{C}$ bond. The most stable of these is gauche-in (Scheme 2). From the acyclic intermediate, scans of the PES revealed essentially no barrier toward the formation of the DA cycloadduct; no saddle point could be found. Two distinct TSs were located corresponding to the formation of the competing 4-membered cycloadduct, the lower of which lies $2.2 \mathrm{kcal} / \mathrm{mol}$ above this intermediate. We also considered the rotation of the exocyclic C-C bond of $5^{+}$, a process which results in the loss of suprafacial stereoselectivity. The barrier height for this rotation is 0.4 $\mathrm{kcal} / \mathrm{mol}$. Structures and spin density plots are shown in Fig. S2.

The fate of $5^{+}$. is finely balanced, with barriers of $\sim 0$, 0.4 and $2.2 \mathrm{kcal} / \mathrm{mol}$ with respect to 6 -membered ring formation, $\mathrm{C}-\mathrm{C}$ rotation or 4-membered ring formation. The PES is extremely flat in this region, raising the possibility of non-TST behavior. Based on the IUPAC definition of an intermediate as 'a molecular entity with a lifetime appreciably longer than a molecular vibration (corresponding to a local potential energy minimum of depth greater than $\mathrm{rt})^{\prime},{ }^{30}$ we questioned whether $\mathbf{5}^{+}$. would indeed exist long enough to be considered a bona fide intermediate. To address these questions, and the possible role of non-statistical dynamic effects in the radical cation DA reaction we turned to quasi-classical trajectory calculations. We compare the trajectories of both neutral and radical cationic systems.

Taking the harmonic frequencies and normal modes of TS and gauche-in TS1, a total of 150 initial coordinates and momenta were created using Singleton's Progdyn ${ }^{31}$ and simulated using Born Oppenheimer Molecular Dynamics (BOMD) (Fig. 1). These structures sample a Boltzmann distribution about the TS dividing surface. Trajectories were propagated in both forward and reverse directions until either the reactants are separated by $>5 \AA$ or a cycloadduct is formed (both forming $\mathrm{C}-\mathrm{C}$ bond lengths $<1.6 \AA$ ). A typical trajectory for each reaction is shown in Videos 1 and 2 in the Supporting Information (SI).
We computed pathways for the neutral and radical cation DA reactions of cyclopentadiene and ethylene (Scheme 2). ${ }^{11}$ Houk's seminal contributions demonstrated the absence of diradical intermediates in neutral DA reactions such as this, ${ }^{27,28}$ and accordingly, a single transition structure (TS) exists in which bond formation is concerted. The computed activation energy in DCM solvent is very high, showing that the reaction would require elevated temperatures for product formation $^{2,15}$. Both $\mathrm{C} 1-\mathrm{C} 2$ and $\mathrm{C} 3-\mathrm{C} 4$ bond lengths in structure TS are $2.23 \AA$.

Consistent with earlier computational results for different substrates ${ }^{4}$ we find that the radical cation DA 


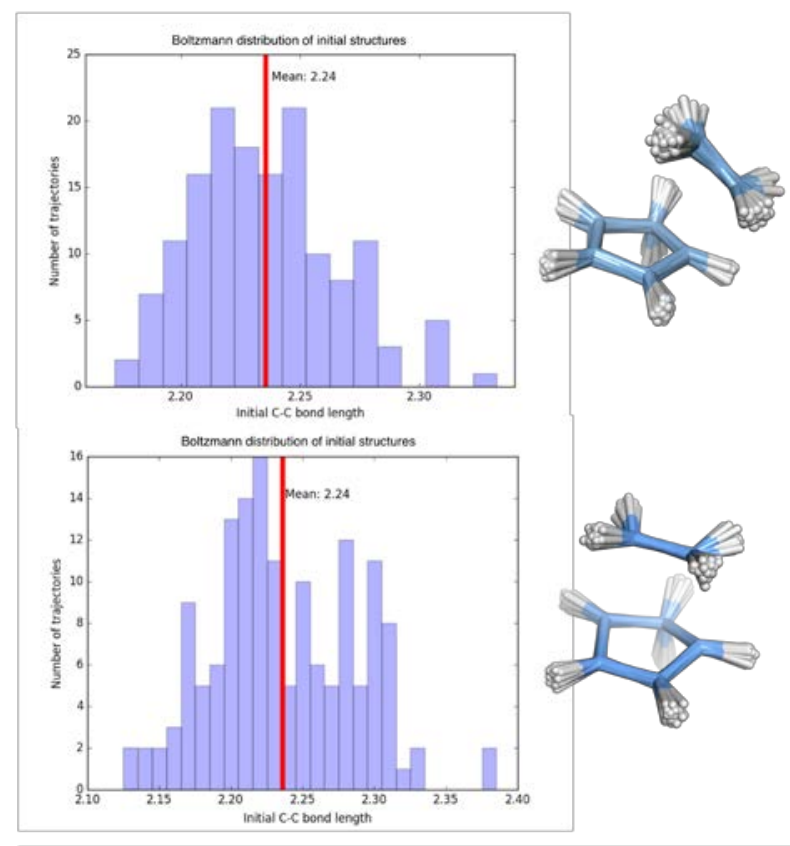

Figure 1. Distribution of forming $\mathrm{C}-\mathrm{C}$ bond lengths for 150 initial structures. Above: radical cation TS, below: neutral TS.

Quasi-classical trajectories are plotted in Fig. 2. Of the 150 trajectories initiated, 142 led to the formation of DA cycloadduct 3; 8 recrossed back to reactants. Of the 150 corresponding radical cation trajectories, the extent of recrossing was more significant. 110 trajectories led to DA cycloadduct $3^{+}$, with none proceeding directly to $4^{+}$. A handful (4) of trajectories "roamed" for a significantly longer time (>1000 fs). 40 trajectories recrossed back to reactants, showing that in asynchronous reactions, recrossing can be substantial. ${ }^{32}$
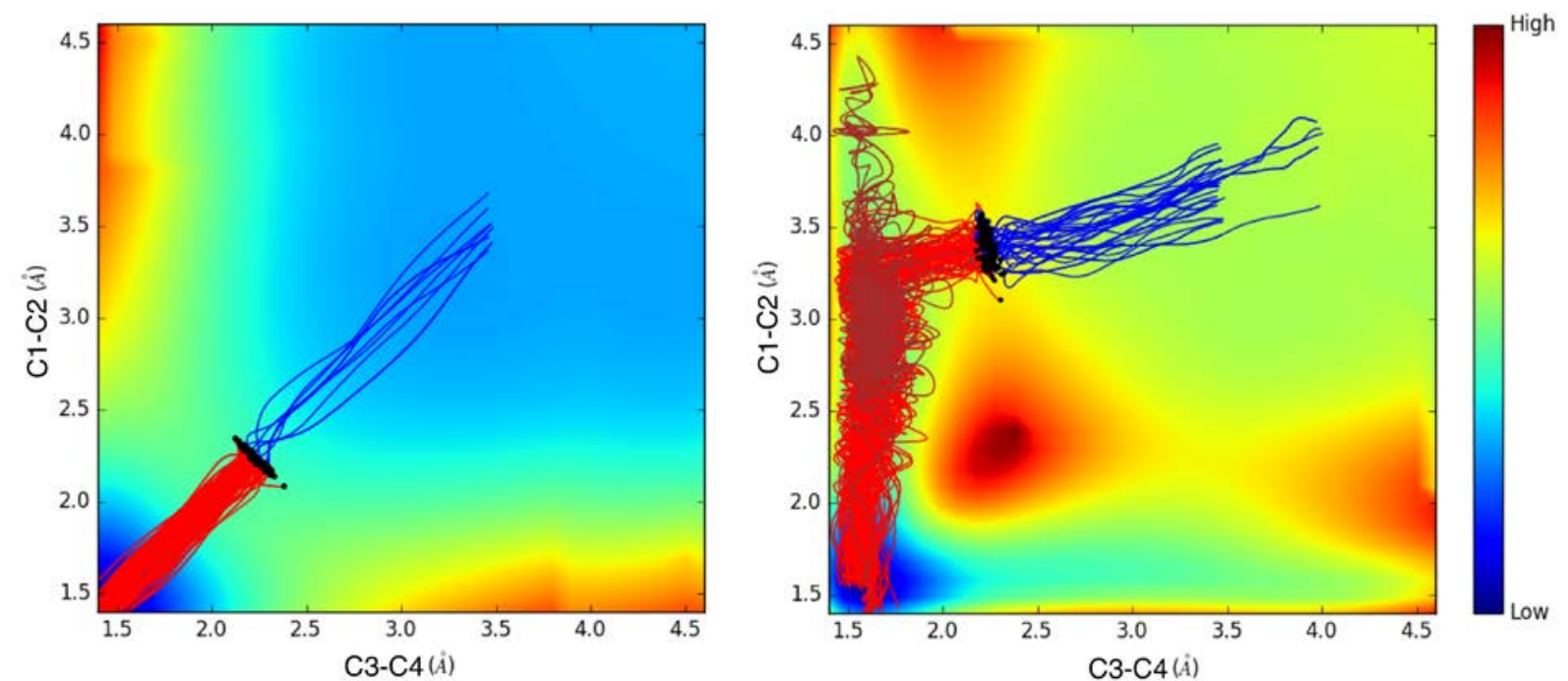

Figure 2. Energy contour plots $(\mathrm{kcal} / \mathrm{mol})$ for neutral $(\mathrm{LHS})$ and radical cation (RHS) DA reactions, with the results of 150 trajectory simulations. Blue paths recross, red and brown paths form the cycloadducts. Black dots are starting structures.

The extent to which these reactions may be considered as either stepwise or concerted can be viewed through the time gaps between the $\mathrm{C}-\mathrm{C}$ bond formation. Houk has introduced the concept of "dynamic concert"15 to describe reactions for which this time gap is less than the lifetime of a C-C bond stretch (30-60 fs). Reactions with a larger time gap may be categorized as dynamically stepwise. In our case, we also consider the timescale of $\mathrm{C}-\mathrm{C}$ bond rotation to be a key quantity in governing suprafacial stereoselectivity. The rotational barrier for the ethyl radical is extremely small (0.4 $\mathrm{kJ} / \mathrm{mol})^{33}$, and so a suitable lower bound for the half-life of this rotation may be estimated from the TST preexponential factor at rt, of 110 fs. Using our computed barrier of $0.4 \mathrm{kcal} / \mathrm{mol}$, we obtained a value of $220 \mathrm{fs}$. We expected that for time gaps substantially longer than these values, C-C rotation will be competitive with cyclization

Using a $1.6 \AA$ cutoff for $\mathrm{C}-\mathrm{C}$ bond formation, the time gap between formation of the first and second bond is shown for both systems in Fig. 3. For the neutral reaction, the average times to form both bonds are around 50 fs (Fig. S3) with an average time gap less than 2 fs. This reaction is dynamically concerted. In contrast, the radical cation DA reaction shows a much greater gap and longer bond formation times (Fig. S4) and more extreme outliers which take much larger times to cyclize. Accordingly, this reaction is dynamically stepwise. The median time gap between bond formation is $\sim 167 \mathrm{fs}$. The average time taken for cyclization is $\sim 365$ $\mathrm{fs}$, with a range of bond formation times between $200 \mathrm{fs}$ and 2700 fs (Fig. S4). Nearly 10\% of the trajectories 
take a long time (>350 fs) for bond rotation to occur between forming the first $\mathrm{C}-\mathrm{C}$ bond $(d 1)$ and the second C-C bond (d2), with 4 trajectories taking more than 1000 fs to form $d 2(\sim 3 \%)$.

The dynamically stepwise nature of the radical cation DA found from these trajectories is somewhat surprising given the absence of a discernible barrier for cyclization of intermediate $\mathbf{5}^{+}$. According to energetic criteria, this species does not constitute an intermediate (the exit barrier is barrier less than rt), however, the changes in bonding are clearly dynamically separated. These results reiterate Singleton's discovery of "dynamic intermediates" for which the energetic definition takes less importance. ${ }^{34}$ The absence of the 4-membered cycloadduct $\mathbf{4}^{+}$. in these trajectories is consistent with the higher barrier towards this product by $2.2 \mathrm{kcal} / \mathrm{mol}$. The time gap certainly allows for several $\mathrm{C}-\mathrm{C}$ bond stretching vibrations (30-60 fs), but what about C-C bond rotation? Indeed, we did observe this occurring in those trajectories which extended beyond 1000 fs.
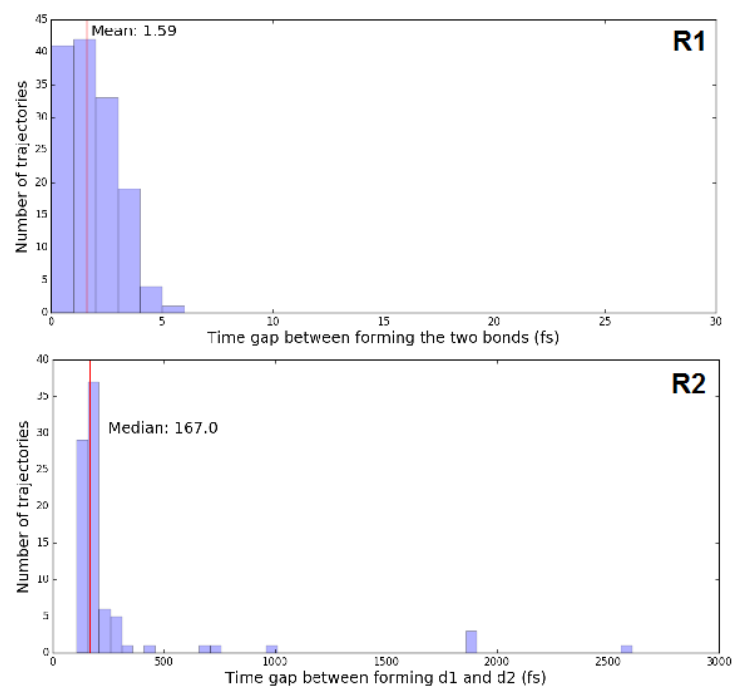

Figure 3. Time gaps between bond formation for neutral DA (R1) and radical cation DA (R2). Note the extended $\mathrm{X}$-axis of the latter.

For the radical cation DA reaction, we compare the results of a typical trajectory (time gap $285 \mathrm{fs}$ ) with a much longer (1976 fs) in Fig. 4 (SI Video 2 vs. Video 3). In the shorter trajectory, $d 1$ forms, and then undergoes several vibrations as motion occurs along $d 2$ towards cycloadduct $3^{+}$. In this second stage, the shortening of the second $\mathrm{C}-\mathrm{C}$ bond is continuous and there is no appreciable time spent in the acyclic conformation. Motion along the two $\mathrm{C}-\mathrm{C}$ forming coordinates is uncorrelated. As shown in Fig. 2, this shape of trajectory is most commonly observed. However, in the longer trajectory, most of the time is spent in the acyclic conformation, from which finally the second $\mathrm{C}-\mathrm{C}$ bond is formed. There are thus two distinct types of trajectory: those which undergo cyclization immediately after the first bond is formed, taking around 170 fs on average, and those in which the acyclic intermediate is present for $>500$ fs.

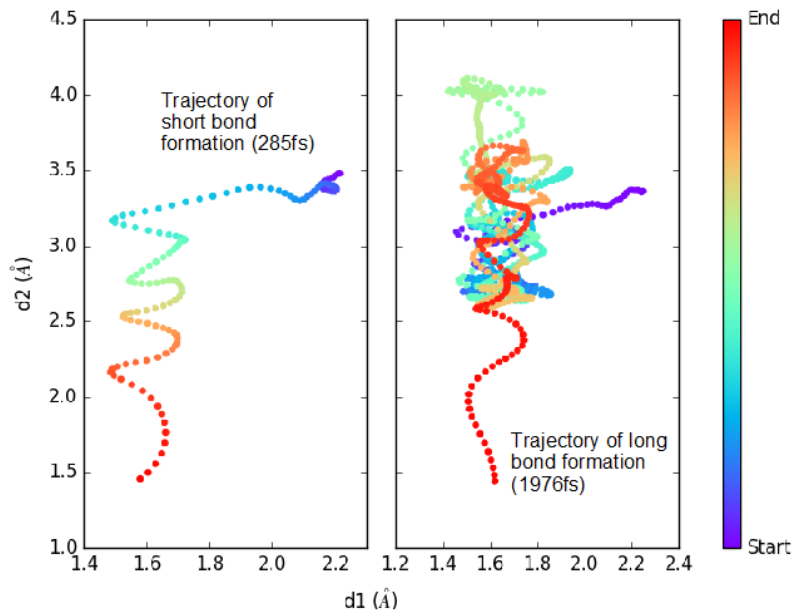

Figure 4. Radical cation DA adduct $\mathbf{3}^{+}$. formation. Comparison of short and long trajectories.

Using distance-based criteria, we were able to detect which trajectories underwent $\mathrm{C}-\mathrm{C}$ bond rotation prior to cyclization (Fig. S5 and Video 3). This was the case for all 4 long trajectories $>1000 \mathrm{fs}$. In light of the above values for the rotation half-life of $220 \mathrm{fs}$, this is to be expected. In shorter trajectories, the second bond begins to form, preventing this rotation (Fig. S6-S8). The loss of stereoselectivity in around $3 \%$ of trajectories, would be experimentally detectable, and lies in between the two extremes of cis- (34\% rotation) and trans-anethole $(0 \%$ rotation). It is plausible that an increase in the rotational barrier will suppress the loss of stereoselectivity, even with long, roaming trajectories ${ }^{35}$ (and vice-versa). The postulate of different mechanisms for these substrates is unnecessary.

In conclusion, quasi-classical dynamics simulations show the existence of a dynamic intermediate in the radical cation DA reaction. The reaction is dynamically stepwise. The time gap between formation of each C-C bond is longer than a molecular vibration (167 \pm 42 fs for $90 \%$ of trajectories, excluding the long ones), but too short to permit C-C bond rotation, which was found to occur in trajectories only after $1000 \mathrm{fs}$. This time gap is 2 orders of magnitude longer than for neutral DA reactions calculated at the same level with solvent. The PES of the radical cation reaction does not predict a barrier for TS2, but nonetheless there is a lifetime to traverse the surface to the product. The "long tail" of trajectories up to 3000 fs permits loss of stereochemistry by rotation. The timescale for this is $-1000 \mathrm{fs}$, allowing the reaction to be dynamically stepwise.

Furthermore, recrossing was found to contribute substantially to the overall frequency of radical cation cycloadditions: ${ }^{31} 27 \%$ of all trajectories recross back to reactants, while only $5 \%$ of trajectories recross in the neutral DA reaction. The competition between $\mathrm{C}-\mathrm{C}$ formation and rotation is fundamental to understanding stereoselectivity in stepwise cyclizations and is the subject of ongoing investigation.

\section{Corresponding author}


Robert Paton [robert.paton@colostate.edu]

\section{Acknowledgment}

This paper is dedicated to Prof. Ken Houk, UCLA on the occasion of his 75th birthday. We acknowledge the Agency for Science, Technology and Research $(A * S T A R)$ Singapore for the National Science Scholarship (NSS) and the EPSRC Centre for Doctoral Training in Theory and Modelling in Chemical Sciences (EP/L015722/1).

\section{Supporting Information Available}

Cartesian coordinates, absolute energies, and imaginary frequencies of all transition structures reported. Gibbs energy profiles, scripts and BOMD settings are also included (PDF). This material is available free of charge via the Internet at http://pubs.acs.org.

\section{References}

(1) Bellville, D. J.; Bauld, N. L.; Pabon, R.; Gardner, S. A; J. Am. Chem. Soc. 1983, 105, 3584-3588.

(2) Bauld, N. L.; Bellville, D. J.; Pabon, R.; Chelsky, R.; Green, G. J. Am. Chem. Soc. 1983, 105, 2378-2382.

(3) Pabon, R. A.; Bauld, N. L. J. Am. Chem. Soc. 1984, 106, 1145-1146.

(4) Wiest, O.; Steckhan, E.; Grein, F. J. Org. Chem. 1992, 57 , 4034-4037.

(5) Valley, N. A; Wiest, O. J. Org. Chem. 2007, 72, 559-566.

(6) Sevov, C. S.; Wiest, O. J. Org. Chem. 2008, 73, 79097915.

(7) Saettel, N. J.; Wiest, O.; Singleton, D. A.; Meyer, M. P. J. Am. Chem. Soc. 2002, 124, 11552-11559.

(8) Sun, L.; Hase, W. L.; Song, K. J. Am. Chem. Soc. 2001 123, 5753-5756.

(9) Lin, S.; Padilla, C. E.; Ischay, M. A.; Yoon, T. P. Tetrahedron Lett. 2012, 53, 3073-3076.

(10) Lin, S.; Ischay, M. A.; Fry, C. G.; Yoon, T. P. J. Am. Chem. Soc. 2011, 133, 19350-19353.

(11) Haberl, U.; Wiest, O.; Steckhan, E. J. Am. Chem. Soc. 1999, 121, 6730-6736.

(12) Bauld, N. L.; Yang, J. Org. Lett. 1999, 1, 773-774.

(13) Bauld, N. L.; Gao, D. J. Chem. Soc. Perkin Trans. 22000 , No. 5, 931-934.

(14) Mann, D. J.; Hase, W. L. J. Am. Chem. Soc. 2002, 124 3208-3209.

(15) Black, K.; Liu, P.; Xu, L.; Doubleday, C.; Houk, K. N. Proc. Natl. Acad. Sci. 2012, 109, 12860-12865.

(16) Hase, W. L.; Kakhiani, K.; Lourderaj, U.; Hu, W.; Birney, D. J. Phys. Chem. A 2009, 113, 4570-4580.

(17) Hare, S. R.; Tantillo, D. J. Pure Appl. Chem. 2017, 89, 679-698.

(18) Ess, D. H.; Wheeler, S. E.; lafe, R. G.; Xu, L.; CelebiÖlçüm, N.; Houk, K. N. Angew. Chem. Int. Ed. 2008, 47, 7592-7601.

(19) Carpenter, B. K.; Harvey, J. N.; Orr-Ewing, A. J. J. Am Chem. Soc. 2016, 138, 4695-4705.

(20) Hare, S. R.; Tantillo, D. J. Chem. Sci. 2017, 8, 1442-1449.

(21) Hare, S. R.; Tantillo, D. J. Beilstein J. Org. Chem. 2016, 12, 377-390.

(22) Hare, S. R.; Pemberton, R. P.; Tantillo, D. J. J. Am. Chem. Soc. 2017, 139, 7485-7493

(23) Hase, W. L.; Song, K.; Gordon, M. S. Comput. Sci. Eng. 2003, 5, 36-44.

(24) Paranjothy, M.; Sun, R.; Zhuang, Y.; Hase, W. L. Wiley Interdiscip. Rev. Comput. Mol. Sci. 2013, 3, 296-316.
(25) Yu, P.; Chen, T. Q.; Yang, Z.; He, C. Q.; Patel, A.; Lam, Y.; Liu, C.-Y.; Houk, K. N. J. Am. Chem. Soc. 2017, 139, 8251-8258.

(26) Grayson, M. N.; Yang, Z.; Houk, K. N. J. Am. Chem. Soc. 2017, 139, 7717-7720.

(27) Houk, K. N.; Loncharich, R. J.; Blake, J. F.; J, W. L. J. J. Am. Chem. Soc. 1989, 84, 9172-9176.

(28) Houk, K. N.; Li, Y.; Evanseck, J. D. Angew. Chem. Int. Ed. 1992, 31, 682-708.

(29) Jorgensen, W. L.; Lim, D.; Blake, J. F. J. Am. Chem. Soc. 1993, 115, 2936-2942.

(30) IUPAC Compend. Chem. Terminol. 2014, 1077, 3096.

(31) Ussing, B. R.; Hang, C.; Singleton, D. A. J. Am. Chem. Soc. 2006, 128, 7594-7607.

(32) Oyola, Y.; Singleton, D. A. J. Am. Chem. Soc. 2009, 131, 3130-3131.

(33) Yamada, K.; Kawashima, Y.; Tachikawa, M. J. Chem. Theory Comput. 2014, 10, 2005-2015.

(34) Yu, P.; Chen, T. Q.; Yang, Z.; He, C. Q.; Patel, A.; Lam, Y.; Liu, C.-Y.; Houk, K. N. J. Am. Chem. Soc. 2017, 139, 8251-8258.

(35) Townsend D.; Lahankar, S. A.; Lee, S. K.; Chambreau, S. D.; Suits, A. G.; Zhang, X.; Rheinecker, J.; Harding, L. B. ; Bowman, J. M. Science 2004, 306, 1158-1161. 